\title{
Étude des phénomènes de transfert de métaux lourds dans une smectite
}

C. POTHIER

A. JULLIEN

C. PROUST

P. LECOMTE

ESEM, Université d'Orléans 45072 Orléans Cedex 2

Chantal.Proust@

univ-orleans.fr

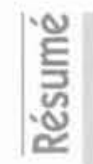

Les argiles gonflantes compactées utilisées comme barrières ouvragées de fond de stockages de déchets urbains, doivent prouver leur efficacité en terme de rétention de polluants tout en respectant la réglementation en matière de perméabilité. Afin d'aborder les problèmes inhérents à la prédiction du comportement à long terme de ces matériaux, deux axes d'étude ont été abordés successivement : i) la réalisation d'essais d'infiltration à pression constante de solutions polluantes modèles (nitrate de cuivre) permettant de déterminer les paramètres du transfert de masse en conditions polluées, ii) l'utilisation d'un code de calcul du transfert de masse en phase liquide pour prédire les durées de saturation d'un massif pollué avec différentes concentrations en nitrates de cuivre. Les résultats expérimentaux, obtenus pour l'argile Fo-Ca, ont montré qu'un échange cationique s'était produit engendrant des modifications tant au niveau de la microstructure de l'argile, du volume de la phase poreuse que de son comportement macroscopique, mais que même pour de fortes concentrations en polluant, l'aptitude au gonflement de la Fo-Ca n'était pas totalement inhibée. Les simulations de la réponse à l'infiltration dans un massif d'argile gonflante en conditions polluées ont mis en évidence la très forte diminution du temps de saturation en surface du stockage.

Mots clés: couplaqe chimio-hydromécanique, nitrate de cuivre, perméabilité, argile gonflante, transfert de masse.
NDLE: Les discussions sur cet article sont acceptées jusqu'au $1^{\text {er }}$ novembre 2003
Expansive compacted clays are often used as engineered barriers for domestic waste cisposals. They must prove their efficiency in terms of pollutant retention while French law concerning maximum allowed permeability has to be achieved. In order to examine the problem related to the prediction of the long term behaviour of such materials, two kinds of analysis were successively performed i) soaking experiments at constant pressure with pollutant model solution (copper nitrate) were performed in order to determine mass transfer parameters under polluted conditions, ii) finite element analysis of mass transfer to predict saturation time of a medium, already polluted, with different concentration of copper nitrates solutions. The experimental results, obtained with the $\mathrm{Fo}-\mathrm{Ca}$ clay, showed that a cationic exchange has occurred, inducing changes in microstructure, in porous phase volume and in macroscopic behaviour. Therefore, even for high pollutant concentration, the swelling potentiel of Fo-Ca was not fully inhibited. The water soaking simulations through a swelling clay medium, pointed out the strong decrease of the saturation time near the clay barrier.

Key words : chemo-hydro-mechanical coupling, copper nitrate. permeability, swelling clay, mass transfer. 


\section{Introduction}

Les sols argileux sont couramment utilisés comme barrières passives ou actives dans les centres d'enfouissement techniques recevant les déchets ménagers, dans le but de ralentir et de limiter la diffusion de polluants issus de la dégradation des déchets vers le sol et les nappes phréatiques. La loi française impose actuellement de respecter une valeur maximale de perméabilité de $10^{-9} \mathrm{~m} / \mathrm{s}$ en fond de décharge. La perméabilité est donc un paramètre conservatif permettant l'évaluation de la sûreté d'un stockage de surface. En fond d'alvéole de stockage, le sol est compacté in situ par temps sec, afin d'optimiser la perméabilité en surface. Par la suite, lors de l'exploitation du site, si aucun géotextile n'est déposé en fond d'alvéole de stockage, ou si ce géotextile est perforé, l'infiltration de l'eau ayant percolé au travers des déchets dans l'argile (lixiviats) devient possible. Ces lixiviats peuvent contenir différents éléments dont certains sont toxiques, essentiellement les métaux lourds en solution, et indésirables pour les nappes phréatiques. Dans le cas oủ l'argile constituant la barrière ouvragée est gonflante, c'est-àdire d'un grand intérèt car faiblement perméable, un problème supplémentaire celui de son rôle en tant que barrière active susceptible de piéger des polluants, est à étudier. Durant la mise en place de la barrière, puis la mise en service du stockage, le degré de saturation augmente au fur et à mesure de l'infiltration par le sol. Dans le cas de l'infiltration d'un lixiviat en provenance de la surface, des réactions physico-chimiques produites lors du contact de l'argile sont susceptibles de modifier les caractéristiques microstructurales de cette dernière et donc la cinétique de transfert de masse macroscopique. De plus, dans le cas du stockage des déchets municipaux, le ruissellement de l'eau de pluie, associé à la dégradation exothermique des déchets et aux variations climatiques, peut conduire à l'infiltration d'une mixture biogaz-lixiviat, ce qui ajoute à la complexité du problème à analyser

Ainsi, les performances de confinement de la barrière argileuse liees au ralentissement du transfert des polluants doivent être garanties; quels que soient les effets couplés entre les transferts de masse et de chaleur, la chimie de l'eau interstitielle et les contraintes appliquées.

Dans le cadre d'études de sites rẻalisées en France, Pronost et Marejka (2000), et au Maroc, Hakkou et al. (2000), font état de la composition de lixiviats recueillis en fond de décharge, dans lesquels on constate généralement que le $\mathrm{pH}$ varie entre 4,5 et 7 pour des lixiviats jeunes (de moins de cing ans) et entre 7 et 9 pour des lixiviats stabilisés (de plus de 10 ans). Les analyses réalisées sur ce type de lixiviat ont montré la présence de différents ions métalliques tels que le zinc, le plomb ou encore le cuivre. Selon Mertens et al. (1995), la concentration en cuivre dans certains lixiviats de décharge de déchets ménagers et assimilés peut atteindre $5,4.10^{-3} \mathrm{~mol} / \mathrm{l}$. Bien que ce polluant ne soit pas le plus toxique, son aptitude à s'échanger avec les ions alcalins et alcalino-terreux contenus dans l'argile et sa grande mobilité comparativement à d'autres métaux est bien connue (Wu et Li, 1998).

Par ailleurs, des études portant sur les mécanismes de diffusion d'ions radioactifs (Kozaki et al., 1999), de cations alcalins et alcalino-terreux (Shainberg et al., 1987; Rytwo et al., 1996; Cases et al., 1997) et de cations métalliques polluants tels que $\mathrm{Pb}^{2+}$ (Delattre-louvel et al., 1993), Ni2* (Singhal et al., 1976), Zn²- (Brandl, 1992) ont montré la capacité des montmorillonites calciques ou sodiques à la rétention des polluants. D'ailleurs, il est admis dans la littérature que le gonflement, la perméabilité et également la compressibilité des argiles sont liés aux caractéristiques microscopiques telles que : - la composition minéralogique de l'argile :

- la valence des cations et la capacité d'échange cationique (Bradbury et Baeyens, 1999) :

- la structure à l'échelle atomique (orientation des feuillets de l'argile) :

- la texture à l'échelle des agrégats de par la distribution des pores et des grains (Benzel et Graf, 1984; Veniale, 1985).

Dans ce contexte, ce travail avait pour objectif l'étude des effets couplés chimio-hydromécaniques produits lors de la percolation de solutions contenant des métaux au travers d'une argile gonflante et d'en évaluer les conséquences sur ses propriétés de confinement. Une étude expérimentale a d'abord été réalisée pour mettre en évidence les phénomènes et résultats significatifs. Afin de contròler les paramètres de l'étude et de mette en évidence les effets produits, la Fo-Ca une smectite calcique fortement gonflante et des solutions de nitrates de cuivre de concentration croissante, préparées en laboratoire, ont été utilisées. Deux techniques d'injection de ces solutions dans l'argile ont été successivement mises au point, permettant d'injecter soit un mélange air/solution soit la solution seule. Les comportements chimio-hydromécanique de l'argile à court terme et à moyen terme ont ensuite été analysés d'après les mesures à différentes échelles macroscopique et microscopique. Puis, les paramètres nécessaires à la simulation du transfert de masse en phase liquide au travers de l'argile ont été déterminés après l'injection des polluants. Une étude prédictive du transfert de masse d'eau dans un massif de Fo-Ca polluée a ensuite été effectuée pour mettre en évidence l'évolution des temps de transfert selon différentes concentrations de polluant.

\section{2}

\section{Étude expérimentale du transfert de masse}

\section{1}

\section{Dispositifs d'injection des solutions}

Les dispositifs développés pour réaliser ce type d'essais comportent deux éléments principaux qui sont la cellule œdométrique et le système d'injection (Fig. 1). Le problème à résoudre concernant l'injection de solutions polluées se posait dans les termes suivants :

- nécessité d'injecter des solutions chimiquement agressives dont la nature corrosive conduirait à la détérioration du corps de pompe dans le cas de l'adaptation d'une technologie classique du commerce :

- nécessité de contrôler avec une très grande précision les volumes de solutions entrant dans l'argile ce que n'aurait pas forcément permis une pompe comme celle utilisée pour le contrôle de la mise en charge du piston de la cellule. 
D'où, des contraintes de conception qui ont été résolues en développant une technique d'injection spécifique.

Les cellules œdométriques (Fig. 1, repère A) d'un diamètre de $40 \mathrm{~mm}$, ont été usinées en matériau inoxydable, afin de pouvoir résister aux solutions chimiquement agressives qui sont injectées dans les échantillons d'argile et à l'argile elle-même lorsque sa capacité d'échange est importante. Les cellules sont équipées en partie basse d'un piston pour contrôler la contrainte mécanique axiale imposée à l'échantillon. Les déplacements du piston sont mesurés avec un comparateur. Lorsque le piston est ramené en position basse la hauteur totale de la cellule est de $40 \mathrm{~mm}$. Des disques poreux en bronze surmontés d'un anneau en téflon sont disposés en haut et en bas de la cellule pour permettre le passage du liquide et de l'air au travers de l'échantillon.

La conception et la mise au point des deux systèmes d'injection se sont déroulées en deux étapes conduisant à la fabrication de deux prototypes. Un premier dispositif appelé $\approx$ cedomètre à réservoir $»$ (Fig. 1, repères $\mathrm{A}$ et B) a êté développé et la faisabilité des essais démontrée. Puis afin de mieux contrôler les conditions d'injection, notamment les mesures du volume de solution entrant dans l'argile, un deuxième dispositif désigné par « cedomètre à seringue » (Fig. 1, repères A et C) a été finalisé (Jullien et Lecomte, 2000). Ces deux systèmes d'injection sont respectivement caractérisés par: - ádomètre à réservoir : système permettant de réaliser l'injection d'un mélange d'air et de solution puisque de l'air comprimé à pression contrôlée est directement mis en contact avec la solution ;
- codomètre à seringue : système permettant d'effectuer seulement l'injection de la solution au moyen d'un piston.

D'où, ces deux systèmes permettent de simuler un ensemble de conditions d'infiltration représentatives des conditions in situ de stockage de déchets municipaux. Le réservoir et la seringue, usinés dans des matériaux transparents et chimiquement inertes, sont instrumentés de manière à permettre la mesure du volume injecté. Cette mesure est obtenue après lecture directe de la hauteur de solution sur une échelle graduée sur la paroi extérieure du réservoir ou après lecture sur un comparateur des déplacements du piston de la seringue.

Ainsi, quelle que soit la technique d'injection, la pression d'injection de la solution est contrôlée séparément de la contrainte mécanique appliquée à l'échantillon d'argile ce qui donne une souplesse supplémentaire dans l'utilisation de ces appareils.

\section{2}

\section{Matériau et solutions}

Le matériau utilisé pour cette étude, l'argile gonflante Fo-Ca est une argile naturelle contenant $80 \%$ de smectite dont les compositions chimiques (exprimée en pourcentage en masse d'oxyde correspondante) et minéralogique sont reportées dans le tableau I. Ses propriétés géotechniques, indiquent qu'il s'agit d'une argile très plastique, avec une limite de plasticité de $52 \%$ et une limite de liquidité de $120 \%$. Les solutions retenues pour l'infiltration sont : soit de l'eau pure (eau distillée), soit des solutions contenant différentes

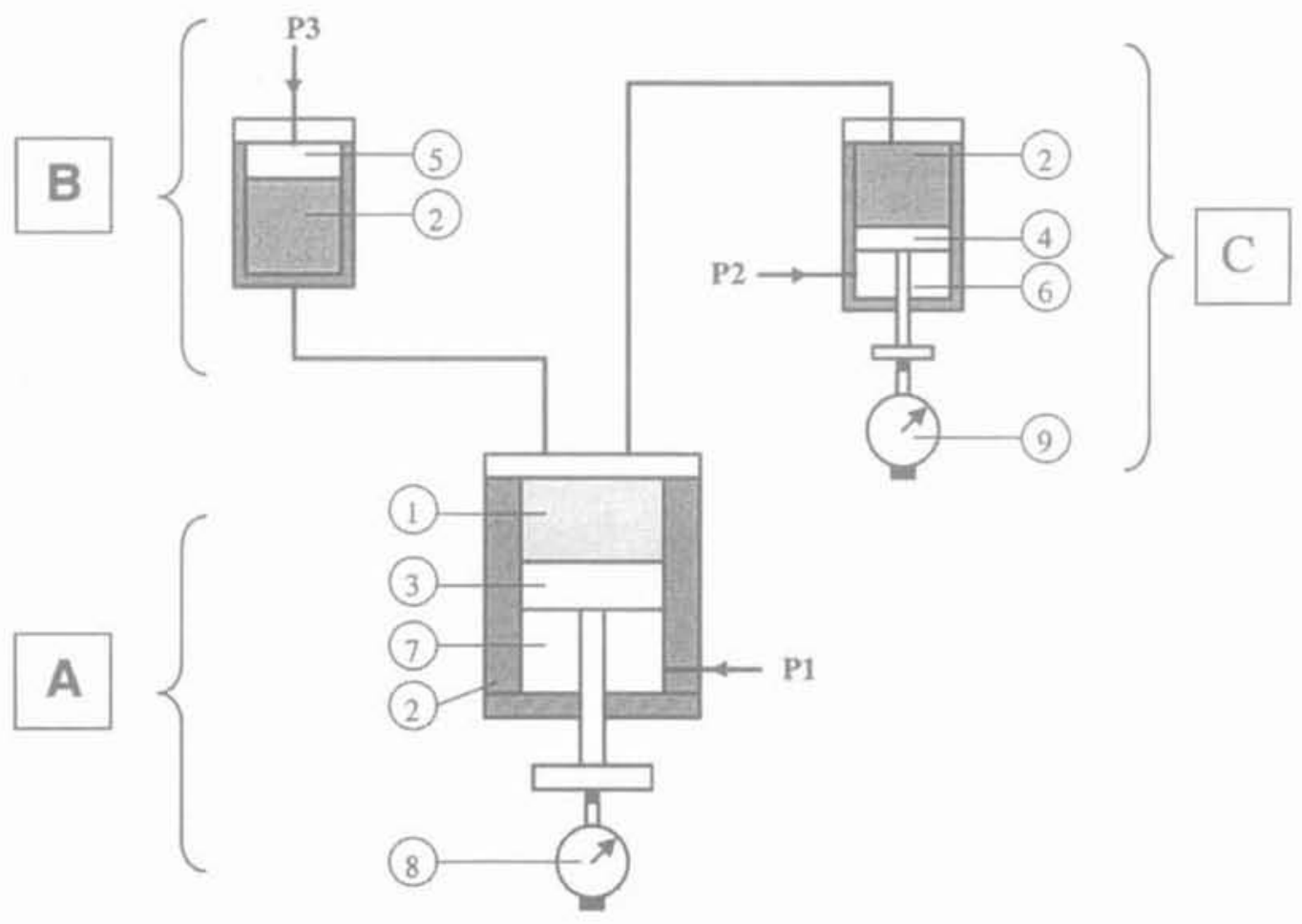

FG.1 Schéma de principe des différents appareillages: (1) argile; (2) solution; (3) piston de la cellule; () piston de la seringue; (5) gaz (P3, pression appliquée pour l'injection du mélange air-solution); (6) gaz (P2, pression appliquée pour l'injection de la solution); (3) gaz (P1, pression de gaz permettant d'appliquer une contrainte mécanique); (8) et () mesure du volume de solution injectée.

Schematic view of the equipment: (1) clay; (2) solute; (3) cell piston; (4) syringe piston; (8) gas (P3, gas pressure for gassolute mixture injection); (6) gas (P2, gas pressure for solute injection); (0) gas (P1, gas pressure for mechanical stress control); () \& (2) dial indicator measure of the injected solute volume. 
TABIEAUI Composition chimique et composition minéralogique de la Fo-Ca.

Chemical and mineralogical composition of $\mathrm{Fo}-\mathrm{Ca}$.

\begin{tabular}{|c|c|c|c|}
\hline \multicolumn{2}{|c|}{ Composition \% } & \multicolumn{2}{|c|}{ Composition minèrale \% } \\
\hline $\begin{array}{l}\mathrm{SiO}_{2} \\
\mathrm{Al}_{2} \mathrm{O}_{3} \\
\mathrm{Fe}_{2} \mathrm{O}_{3} \\
\mathrm{MnO} \\
\mathrm{MgO}\end{array}$ & $\begin{array}{c}44,87 \\
22,33 \\
8,68 \\
<\text { L.D. } \\
0,92\end{array}$ & $\begin{array}{l}\text { argile } \\
\text { quartz } \\
\text { goéthite } \\
\text { calcite } \\
\text { gypse }\end{array}$ & $\begin{array}{c}86,2 \\
6 \\
6 \\
1,4 \\
0,4\end{array}$ \\
\hline $\mathrm{CaO}$ & 2,69 & \multicolumn{2}{|c|}{ Composition argileuse \% } \\
\hline 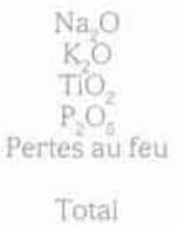 & $\begin{array}{c}0,35 \\
0,16 \\
1,27 \\
0,1 \\
18,43 \\
99,8\end{array}$ & $\begin{array}{l}\text { Smectite } \\
\text { Kaolinite } \\
\text { CEC' meq/100g } \\
\text { Surface spécifiquè } \\
\text { (éthylène glycol) } \mathrm{m} / \mathrm{g}\end{array}$ & $\begin{array}{c}80 \\
20 \\
\\
98 \\
715\end{array}$ \\
\hline
\end{tabular}

- Méthode de Mantin I.. 1969. C.R. Acad. Sc., Paris, 269. 815-818.

"Mćthode d'Eltantawy I.M. Arnoid P.M. 1974. I. Soil Sc. 25, 99.110

concentrations de nitrate de cuivre $\mathrm{Cu}\left(\mathrm{NO}_{3}\right)_{2}$. Les nitrates ont été préférés aux chlorures et aux carbonates car ils sont moins agressifs vis-à-vis des matériaux constituant le corps des cellules cedométriques. Des solutions de nitrate de cuivre ont été préparées aux différentes concentrations suivantes : $10^{-3} 10^{-2}, 3.10^{-2}$ et $10^{-1} \mathrm{~mol} / \mathrm{l}$. Cette gamme de concentrations, couvre une large plage de composition de lixiviats de décharge.

\section{3}

\section{Conditions et procédures d'essais}

Pour être en accord avec les conditions de compactage in situ de la barrière argileuse, dans le cas d'un site technique d'enfouissement de déchets ménagers, une pression de consolidation de $0,5 \mathrm{MPa}$ a été retenue pour ces essais de laboratoire. Le poids volumique initial des galettes de Fo-Ca ainsi compactées est de $12 \mathrm{kN} / \mathrm{m}^{3}$. Des essais préliminaires d'injection d'eau pure à volume constant dans l'argile compactée à l'état de poudre puis entièrement déchargée ont mis en évidence la génération d'une pression de gonflement acceptable. Enfin l'épaisseur des galettes a été minimisée pour permettre de rendre les essais de percolation des solutions aussi courts que possible.

D'où, chaque essai a ensuite été réalisé avec une masse initiale de $10 \mathrm{~g}$ de poudre, d'une teneur en eau initiale de $12,5 \%$. Le compactage a été effectué par paliers de contrainte jusqu'à $0,5 \mathrm{MPa}$ et suivi d'une décharge totale. L'injection de la solution a ensuite été réalisée à pression constante de manière à se placer dans des conditions de stockage conduisant à une infiltration une fois les déchets en place. L'infiltration a donc été imposée avec une pression d'injection constante en face supérieure de la galette d'argile de $0,3 \mathrm{MPa}$. La mesure du volume de solution entrant dans la galette d'argile a été effectuée régulièrement, de même que celle de la déformation axiale de l'argile au cours de la saturation en solution puis tout au long des essais. Parallèlement, le percolat (solution ayant traversé le matêriau argileux), a été récupéré dans des tubes inertes chimiquement. La fréquence d'échantillonnage du percolat a été fixée à 48 heures, de façon à obtenir une quantité suffisante de celui-ci à chaque prélèvement. Il a systématiquement été acidifié à l'acide nitrique puis conservé au réfrigérateur avant d'être analysé par spectrométrie d'absorption atomique pour déterminer l'évolution en fonction du temps des concentrations de différents ions tels que $\mathrm{Cu}^{2+}$ (solution injectée) et $\mathrm{Ca}^{2+}, \mathrm{Na}^{*}, \mathrm{Mg}^{2+}, \mathrm{Fe}^{2+}$, cations compensateurs de l'argile considérée et qui par réactions d'échanges physico-chimiques peuvent être relargués dans les percolats; ces échanges ayant lieu dans l'espace basal de l'argile. De plus, comme les différents cations concernés ont des rayons ioniques et des charges différentes, on peut s'attendre à observer des modifications des distances basales en fonction de la nature et de la quantité d'ions échangés, d'où une étude des distances basales par diffraction des rayons $\mathrm{X}$.

Pour compléter cette approche, à la fin des essais, les galettes saturées en solution cuivrique ont été échantillonnées en camemberts puis dans l'épaisseur. Les échantillons obtenus ont permis de déterminer

- la teneur en eau et la porosité totale en fin d'essai après un passage à l'étuve des échantillons à $105^{\circ} \mathrm{C}$ pendant 24 heures :

- le degré de saturation de l'argile en fonction de la pression de succion en réalisant des isothermes d'adsorption, les échantillons ont ainsi été placés dans des dessiccateurs étanches à humidité relative contrôlée grâce à des solutions salines saturées (humidité relative comprise entre $11 \%$ et $98 \%$ ) ;

- l'évolution de la distance basale de l'argile en fonction de la concentration en cuivre par diffraction des rayons $\mathrm{X}$ entre la face infiltrée et la face opposée, les diagrammes de diffraction ont été enregistrés avec un diffractomètre Siemens D500 doté d'une anticathode au cuivre pour des échantillons préalablement passés à l'étuve à $105^{\circ} \mathrm{C}$ pendant 24 heures puis broyés.

La méthodologie d'essais à conduire a ensuite été mise au point. Une première série d'essais a été réalisée avec l'œdomètre à réservoir pour les différentes solutions préparées. Les résultats ont permis de constater qu'il fallait plusieurs dizaines d'heures de percolation pour obtenir un percolat et que même après $500 \mathrm{~h}$, la concentration en cuivre dans le percolat restait en dessous de la limite de détection de l'appareillage. Par la suite, des essais courts (quelques dizaines d'heures) avec le réservoir et la seringue, puis longs (plusieurs centaines d'heures) uniquement avec la seringue, ont été réalisés pour aborder respectivement :

- l'étude des régimes transitoires et permanents obtenus par effet de couplage chimio-hydromécanique et l'évaluation des paramètres de transfert de masse ; - l'analyse phénoménologique des couplages pour interpréter le comportement macroscopique observé ; - les cinétiques de rétention des métaux polluants par l'argile Fo-Ca.

\section{4}

\section{Étude des effets couplés chimio-hydromécaniques}

\section{9.}

\section{Régimes transitoires et phénomènes observés}

La figure 2a présente les mesures du volume de solution injectée, de la déformation due au gonflement en fonction du temps, pour un essai d'infiltration réalisé avec l'cedomètre à seringue avec une solution de 

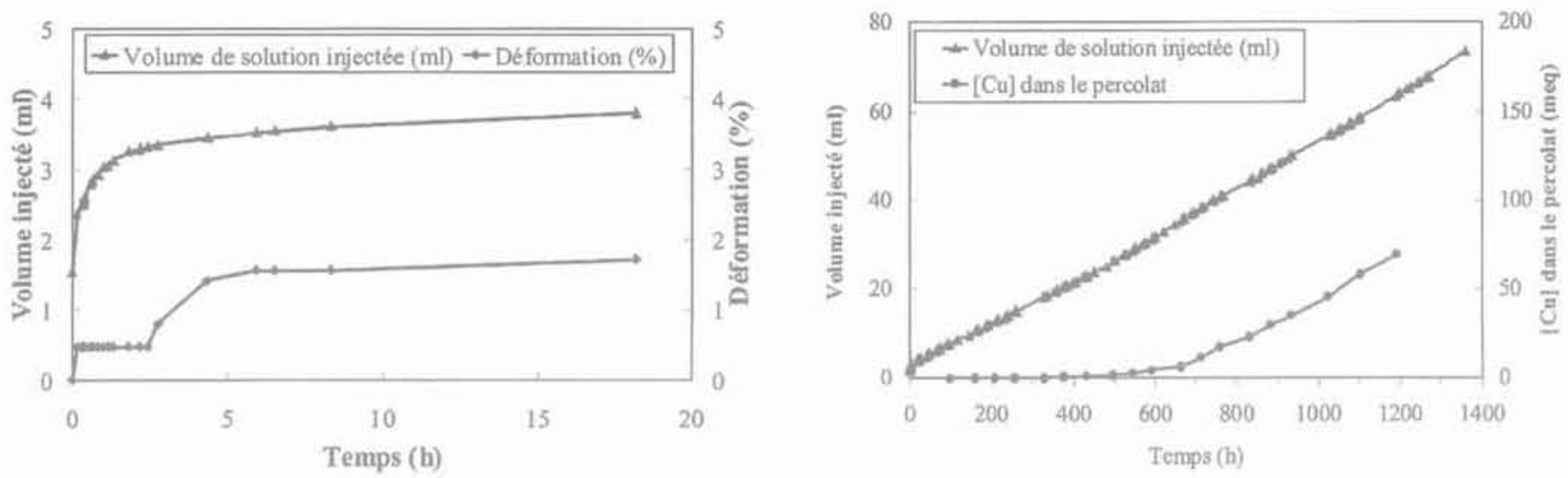

FG. 2 Réponse de l'argile Fo-Ca à l'injection d'une solution à $10^{-1} \mathrm{~mol} / \mathrm{l}$ de nitrate de cuivre en fonction du temps avec l'œdomètre à seringue : a) évolution du volume injecté et de la déformation de gonflement axiale ; b) évolution du volume injecté et de la concentration en cuivre dans le lixiviat.

An example of solute intake with $\left[\mathrm{Cu}^{2+}\right]=10^{-1} \mathrm{~mol} / \mathrm{l}$ versus time using the syringe oediometer; a) evolution of solute intake and swelling axial strain; b) evolution of solute intake and copper concentration in leachate.

nitrate de cuivre de concentration $10^{-1} \mathrm{~mol} / \mathrm{l}$ pendant environ 18 heures. Le gonflement microscopique des premières couches d'argile nécessite une quantité suffisante d'eau pour que le gonflement macroscopique soit mesurable. Ceci se traduisant par un plateau. durant les trois premières heures d'imbibition, sur la courbe de déformation en fonction du temps (Fig. 2a). La déformation et le débit d'injection sont constants au bout de quelques heures, dès la sortie du percolat en bas de l'échantillon. Les régimes permanents mécanique et hydraulique sont donc atteints très rapidement. Durant cet essai, le volume de percolat recueilli n'etait pas suffisant pour permettre une analyse chimique. La figure $2 \mathrm{~b}$ présente les résultats d'un essai réalisé dans les mêmes conditions mais pour un temps beaucoup plus long, d'environ 1360 heures. La courbe de déformation en fonction du temps n'a pas été reportée sur cette figure, du fait qu'aucune variation de déformation n'est observée après 15 h d'injection. La courbe du volume injecté présente une rupture de pente, inhabituelle lors d'une infiltration à l'eau pure, après 450 heures de percolation, qui correspond à une augmentation significative de la concentration en cuivre dans le lixiviat. Ainsi, la perméabilité a une valeur de $2,110^{-12} \mathrm{~m} / \mathrm{s}$ jusqu'à 450 heures, puis augmente et se stabilise à $2,410^{-12} \mathrm{~m} / \mathrm{s}$ entre 450 et 1360 heures. Par ailleurs, les analyses chimiques réalisées sur le percolat obtenu après 48 h d'infiltration n'ont pas révélé la présence de $\mathrm{Cu}^{2+}$ ni même jusqu'à 400 heures d'injection du polluant. Ainsi au début de la percolation tout le cuivre injecté est retenu par l'argile puis progressivement une partie du cuivre se retrouve dans le percolat. On peut estimer qu'il faut attendre plus de trois mois pour que l'argile ne retienne plus du tout le cuivre.

Le cuivre n'étant pas, au début de la percolation, relarqué dans le percolat, il a été nécessairement fixé par la phase solide, ou ralenti à la traversée du squelette argileux. Pour étayer l'une ou l'autre des deux hypothèses, des comparaisons entre face infiltrée et face opposée des galettes ont aussi été réalisées en fin d'essai à partir des spectres de diffractions des rayons X. La figure 3 représente l'évolution de la raie d(001) distance basale de l'argile (Caillère et al., 1982) en fonction de la zone de prélèvement après infiltration d'une solution de nitrate de cuivre pendant 1360 heures. La raie $\mathrm{d}(001)$ de l'argile Fo-Ca avant infiltration est aussi reportée sur cette figure comme référence : elle est symbolisée par une barre verticale positionnée à 5,7 degré pour une abscisse graduée en $2 \theta$ ( $\theta$, angle de diffraction). L.es spectres obtenus après injection de polluant, présentent un décalage de la raie (001) vers les grands angles, par rapport à la position de la raie de l'argile non polluée. Ces résultats indiquent une diminution de la distance basale due au remplacement des ions compensateurs par des cations $\mathrm{Cu}^{2+}$ totalement fixés dans la structure de l'argile. Un décalage plus important est observé pour un échantillon prélevé en face supérieure, ce qui prouve l'existence d'un gradient de concentration en $\mathrm{Cu}^{2+}$ entre le haut et le bas de l'échantillon, gradient observé même pour un essai d'injection du polluant de courte durée. De plus, dès lors que le matériau se trouve en suspension, les échanges cationiques sont quasi instantanés alors qu'ils ralentissent très fortement pour un matériau compactẻ puisque sa surface d'échange avec la solution diminue en fonction du compactage. Ainsi se crée un gradient de concentration entre la face injectée au contact avec la solution entrante et la face opposée. Il est donc clair que si les régimes permanents hydraulique et mécanique s'établissent après quelques heures, le régime "chimique » s'établit longtemps après.

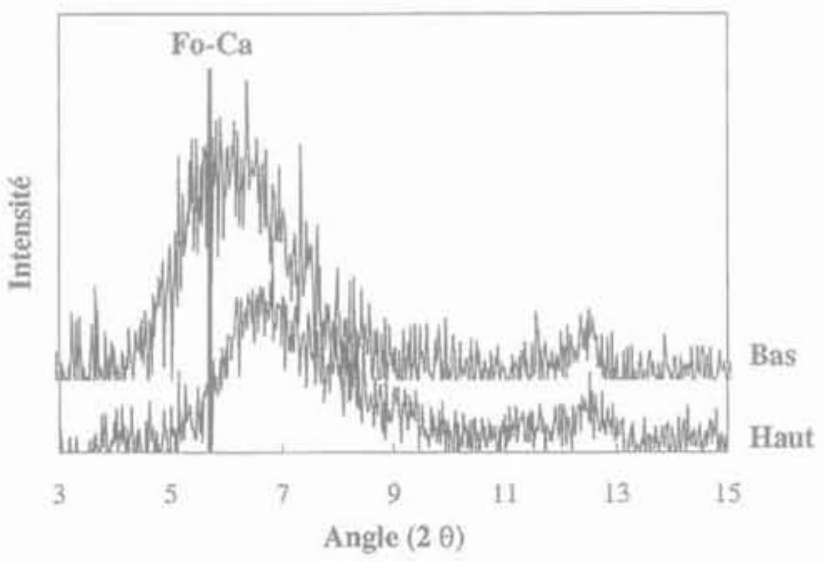

FIG. 3 Évolution des spectres de diffraction des rayons $\mathrm{X}$ de la Fo-Ca après 1360 heures d'infiltration par une solution de nitrate de cuivre à $10^{-1} \mathrm{~mol} / \mathrm{l}$ en fonction de la zone de prélèvement en comparaison avec le spectre de la Fo-Ca matérialisé par une droite d'abscisse 5,7 degré en 20.

Evolution of the $\mathrm{X}$-ray diagrams on Fo-Ca sample soaked with $10^{-1} \mathrm{~mol} / \mathrm{l}$ copper solution during 1,320 hours, at the top and at the bottom in comparison with pure Fo-Ca represented by a line a 5.7 degrees in $2 \theta$. 


\section{Q.9.9.}

\section{Détermination des paramètres du transfert de masse}

Les résultats des essais réalisés ont été exploités dans le cadre d'une approche simplifiée au seul problème du transfert de masse en phase liquide. Les équations du transfert de masse en phase liquide et les différents paramètres associés sont présentés ci-dessous,

Le mouvement de l'eau est gouverné par la loi de Darcy :

$$
\overrightarrow{\mathrm{v}}=-\frac{\mathrm{kr}_{\mathrm{f}}}{\gamma_{\mathrm{f}}}\left(\left[\operatorname{grad}\left(\mathrm{P}_{\mathrm{r}}-\mathrm{P}_{\mathrm{a}}\right)\right]+\rho_{r} \operatorname{ggrad}(\mathrm{z})\right)
$$

avec $: k_{\mathrm{f}}=\mathrm{k}_{\mathrm{s}} \cdot \mathrm{k}_{\mathrm{H}}$

$\vec{v}$ : la vitesse de Darcy $(\mathrm{m} / \mathrm{s})$; $\mathrm{k}_{\mathrm{f}}$ : le coefficient de perméabilité au fluide $(\mathrm{m} / \mathrm{s}) ; \mathrm{P}_{\mathrm{f}}$ : pression du fluide; $\mathrm{P}_{\mathrm{a}}$ : pression atmosphérique ; $\mathrm{z}$ : la cote de l'échantillon: $\mathrm{k}_{\mathrm{ff}}$ : la perméabilité relative et $\mathrm{k}_{\mathrm{s}}$ : perméabilité à saturation $(\mathrm{m} / \mathrm{s}) ; \rho$, masse volumique du fluide : $10^{3} \mathrm{~kg} \cdot \mathrm{m}^{-3}$; $\mathrm{g}$ : accélération de la gravité $\left(\mathrm{m} / \mathrm{s}^{2}\right) ; \gamma_{\mathrm{f}}$ : le poids volumique du fluide : $10^{4} \mathrm{~N} \cdot \mathrm{m}^{-3}$.

Compte tenu que le terme de pression est prépondérant devant celui lié à $z$, on négligera donc par la suite, l'effet de z dans la charge hydraulique.

L'équation de la conservation de masse s'exprime par:

$$
\frac{\partial\left(\rho_{r} n S_{r}\right)}{\partial t}+\operatorname{div}\left(\rho_{r} \vec{v}\right)=0
$$

où $\mathrm{S}_{-}$est le degré de saturation et $\mathrm{n}$ la porosité. obtient :

En reportant l'équation (1) dans l'équation (2), on

$$
\frac{\partial\left(\rho_{r} \mathrm{nS}_{r}\right)}{\partial \mathrm{t}}+\operatorname{div}\left(-\frac{\mathrm{k}_{r}}{\mathrm{~g}} \operatorname{grad}\left(\mathrm{P}_{r}-\mathrm{P}_{\mathrm{a}}\right)\right)=0
$$

A partir du débit obtenu en régime permanent, la perméabilité intrinsèque $\mathrm{K}\left(\mathrm{m}^{2}\right)$ est déterminée d'après:

$$
\mathrm{K}=\frac{\mathrm{k}_{\mathrm{s}} \cdot \mu_{\mathrm{f}}}{\gamma_{\mathrm{f}}}
$$

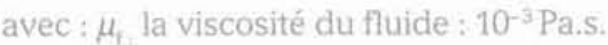

L'exploitation des résultats des mesures précédentes, afin de déterminer un jeu complet de paramètres incluant: la perméabilité, la porosité et l'évolution du degré de saturation en fonction de la pression capillaire, $\mathrm{P}_{\mathrm{c}}=\mathrm{P}_{\mathrm{f}}-\mathrm{P}_{\mathrm{a}}$, est présentée ci-après pour les différentes concentrations de nitrate de cuivre imposées. La figure 4 présente les variations de la perméabilité k pour les deux systèmes d'injection en fonction de la concentration en cuivre dans la solution injectée. Dans les deux cas, la perméabilité de l'argile Fo-Ca augmente lorsque la concentration en cuivre de la solution augmente, et montre une tendance asymptotique pour les fortes concentrations. Par exemple, avec l'œdomètre à seringue, la perméabilité est multipliée par un coefficient 2,2 entre l'injection d'eau pure et de la solution à $10^{-1} \mathrm{~mol} / \mathrm{l}$ de cuivre. De plus, quelle que soit la concentration en cuivre, la perméabilité de l'argile obtenue avec l'œedomètre à réservoir est supérieure d'un ordre de grandeur à celle obtenue avec l'cedomètre à seringue. La figure 5 présente les courbes de rétention d'eau de l'argile Fo-Ca, après pollution au nitrate de cuivre ainsi que celle obtenue pour une argile non gonflante (la kaolinite). L'effet de la pollution se traduit par un décalage de la courbe de rétention vers les faibles degrés de saturation et vers l'origine des pressions capillaires. Cette tendance rapproche la Fo-Ca polluée d'une argile non gonflante et montre qu'il y a bien modification du pouvoir gonflant de l'argile Iors de la percolation du nitrate de cuivre. Enfin, la porosité finale a été déterminée pour chaque concentration (Tableau II) : elle est peu différente pour toutes les concentrations en nitrate de cuivre utilisées.

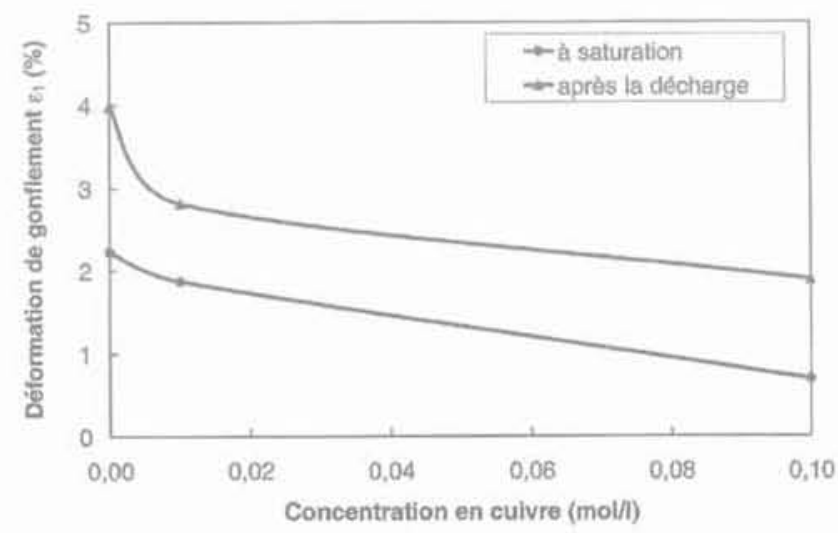

FiG.4 Perméabilité en fonction de la concentration en cuivre dans la solution injectée et en fonction du système d'injection.

Permeability as a function of copper concentration with the two sets of boundary conditions.

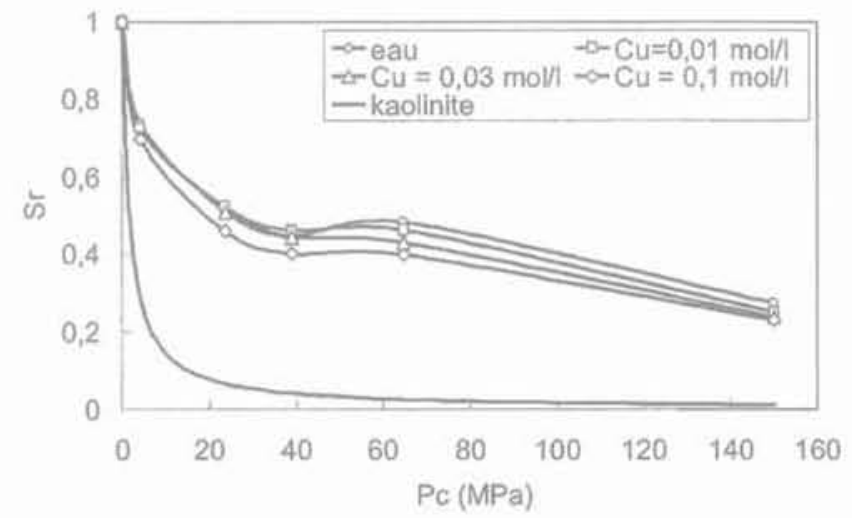

FiG. 5 Courbes de rétention après pollution de l'argile avec des solutions de nitrate de cuivre.

Retention curves of the samples after soaking with various copper concentrations.

TABLEAU II Paramètre d'entrée du modèle en fonction de la concentration de cuivre. Input model parameters versus copper concentration.

\begin{tabular}{l|c|c|c|c}
\multicolumn{1}{c|}{ Concentration en cuivre $(\mathrm{mol} / \mathrm{l})$} & 0 & $10^{-2}$ & $3.10^{-2}$ & $10^{-1}$ \\
\hline Paramètre a & $1,8.10^{-4}$ & $1,9.10^{-1}$ & $2,0.10^{-1}$ & $2,3.10^{-1}$ \\
\hline Perméabilité $(\mathrm{m})$ & $1,08 \cdot 10^{-12}$ & $1,37 \cdot 10^{-12}$ & $1,5710^{-12}$ & $2,3310^{-12}$ \\
\hline Porosité & 0,55 & 0,55 & 0,62 & 0.55 \\
\hline
\end{tabular}




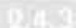

\section{Discussion sur les phénomènes observés}

Pour affiner l'interprétation des résultats précé dents, les mesures de déformation de gonflement au cours de la saturation de l'argile et en fin d'essai après décharge totale du matériau ont été reportées en figure 6 pour les essais avec la seringue. Les tracés, reportés en fonction de la concentration en cuivre montrent que:

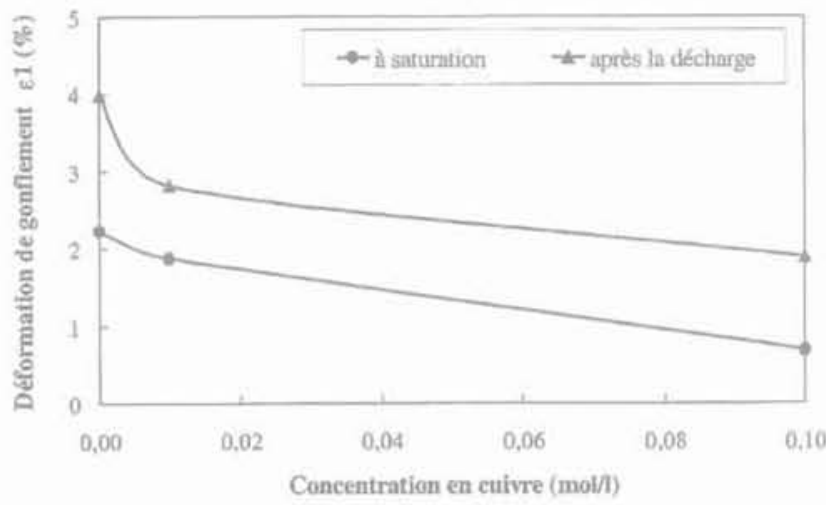

FG. 6 Déformations de gonflement de l'argile en fonction de la concentration en cuivre dans la solution avec l'odomètre à seringue: comparaison entre la déformation obtenue à saturation et celle obtenue après la décharge hydromécanique en fin d'essai. Final swelling strain at saturation as a function of copper concentration with the syringe oedometer, comparison of the swelling strain at saturation and after unloading with respect to copper concentration.

- la déformation du matériau argileux après saturation à l'eau pure est faible de l'ordre de quelques pour cent, ce qui résulte des conditions d'essai (compactage jusqu'à $0,5 \mathrm{MPa}$ puis décharge totale):

- le gonflement par saturation à l'eau pure est trois fois plus élevé qu'avec la solution de cuivre à $10^{-1} \mathrm{~mol} / \mathrm{l}$, ce qui confirme l'inhibition du gonflement par le nitrate de cuivre;

- la courbe d'évolution de la déformation de la Fo-Ca après décharge mécanique en fin d'essai a la mème allure que celle obtenue après saturation en solution mais montre une augmentation du gonflement en décharge : l'inhibition du gonflement est donc partielle.

Une partie de l'écart de perméabilité mesurée après infiltration entre eau pure et nitrate de cuivre peut donc être attribuée à l'inhibition du gonflement lors de la saturation par la solution.

Les différences observées selon la technique d'injection (réservoir et seringue) pourraient s'expliquer en partie par le mode de mise en pression de l'eau pour l'injection. En effet, le gaz piégé dans le liquide contenu dans le réservoir, même en faible quantité, contribue à l'augmentation de la cinétique d'injection et du débit et, par conséquent, influe sur la perméabilité. Par ailleurs, la précision de la lecture du volume de solution entrant dans l'argile avec le réservoir peut entraîner une majoration systématique du volume et donc se cumuler avec l'effet précédent pour conduire aux écarts observés entre les deux dispositifs. Malgré ces différences, les tendances obtenues à concentration de cuivre crois- sante sont les mêmes. Pour s'affranchir d'un couplage supplémentaire et éviter de prendre en compte la compressibilité sur le débit, l'étude a été poursuivie par des essais d'infiltration par la technique de la seringue, afin d'étudier uniquement le transfert en phase liquide.

Afin de compléter la caractérisation du comportement à la percolation de l'argile, la figure 7 récapitule l'évolution du cumul des concentrations des ions $\mathrm{Ca}^{2 *}$, $\mathrm{Na}, \mathrm{Mg}^{2+}$ et $\mathrm{Cu}^{2+}$ relargués, en fonction du temps, dans le percolat. La courbe relative au calcium présente une discontinuité après 500 heures qui coîncide avec celle du cuivre (Fig. 7). En ce point d'inflexion, la concentration en calcium dans le lixiviat (exprimée en meq pour $10 \mathrm{~g}$ d'argile) correspond quantitativement au taux de calcite présente dans le matériau brut, déterminé par une analyse élémentaire préalable (exprimée en \% en masse) (Tableau I). Ainsi il apparait ici que les transferts chimiques obéissent à deux régimes différents : dans un premier temps, une réaction de dissolution de la calcite présente dans le matériau brut se produit : elle contribue à une augmentation de la porosité et surtout de la perméabilité bien après que le gonflement s'est stabilisé. Dans un deuxième temps, des réactions d'échanges cationiques entre le calcium, le sodium et le magnésium structuraux de la Fo-Ca et le cuivre a lieu avec une cinétique très différente de la première réaction chimique. Cet échange a pour effet l'augmentation des interactions inter foliaires (diminution de la distance basale) et contribue ainsi à une rigidification à l'échelle microscopique du matériau qu'il faudrait confirmer par des essais mécaniques à saturation.

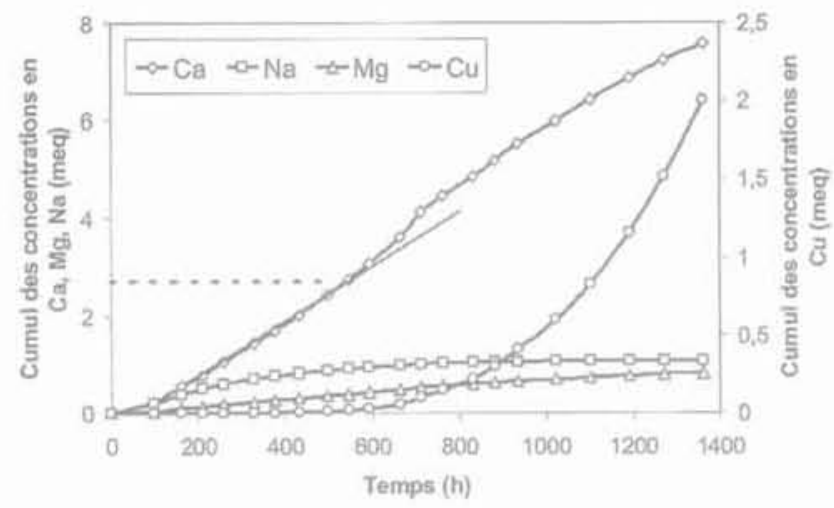

FG.7 Evolution du cumul des concentrations des ions $\mathrm{Ca}^{2+}$ et $\mathrm{Cu}^{2+}$ dans le percolat en fonction du temps.

Evolution in the leachate of $\mathrm{Ca}^{2-}, \mathrm{Na}^{*}, \mathrm{Mq}^{2 *}$ and Cu² cumulative concentrations versus time with a $0.1 \mathrm{~mol} / \mathrm{l}$ copper solution.

\section{3}

\section{Simulation du transfert de masse d'eau dans une argile polluée}

\section{1}

\section{Conditions de simulation et paramètres d'entrée du modèle}

Cette partie présente la modélisation d'un massif argileux non saturé, préalablement pollué, soumis à une re-saturation à l'eau pure. Une telle approche se 

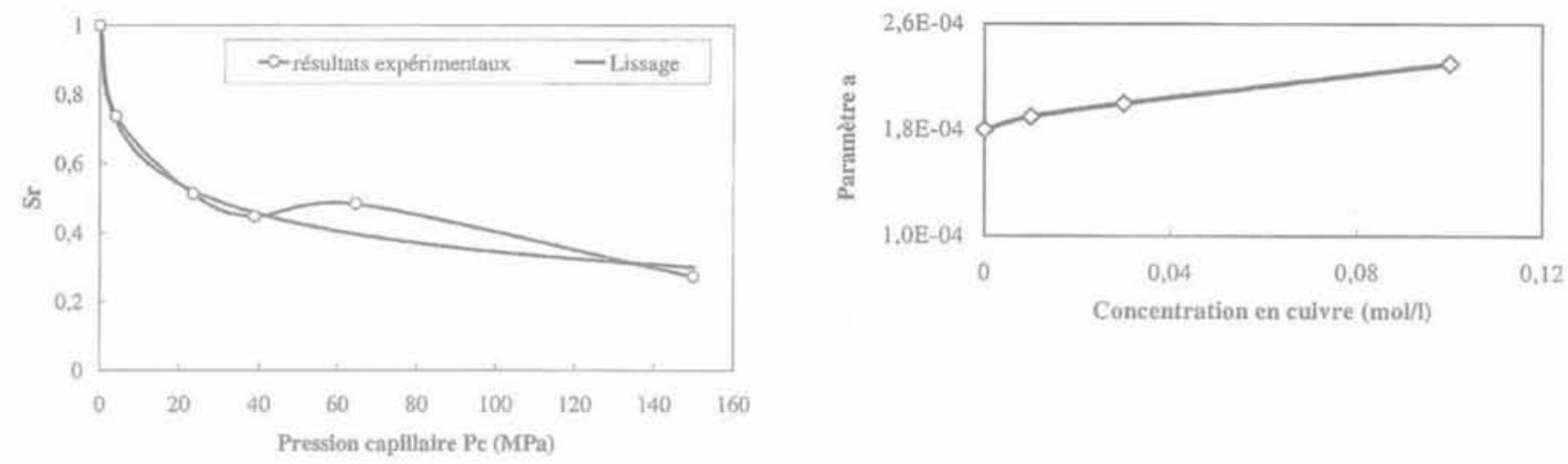

FiG. \& Exploitation des paramètres mesurés pour la Fo-Ca en vue d'une modélisation du transfert de masse : a) exemple de lissage de courbe de rétention par une fonction hyperbolique pour la Fo-Ca infiltrée à l'eau ; b) évolution du coefficient de la loi en fonction de la concentration en cuivre.

Evolution of the Fo-Ca parameters for mass transfer modelling: a) example of retention curve fitted by an hyperbolic function for $\mathrm{Fo}$-Ca soaked with water; b) evolution of coefficient of the $\mathrm{Sr}=\mathrm{f}(\mathrm{Pc})$ curve versus copper concentration.

justifie du fait que les phénomènes transitoires hydromécaniques sont significativement plus courts que les phénomènes transitoires chimiques, bien que la pollution de l'argile au nitrate de cuivre ait des effets très rapides sur les propriétés hydromécaniques du matéríau Fo-Ca. Un découplage des sollicitations a donc été effectué, pour n'aborder que l'étude du transfert de masse en phase liquide, régi par la loi de Darcy (équation [1]) et l'équation de conservation de la masse (êquation 2). Pour les simulations numériques, un code de calcul aux éléments finis du transfert de masse en phase liquide, développé à partir de la formulation variationnelle de l'équation (3) (Pothier et al., 1999; Pothier, 2001) a permis de réaliser les prédictions.

Pour effectuer des simulations sur le matériau FoCa pollué, les paramétres de transfert déterminés lors des essais présentés au paragraphe 2, ont été ré-exploités de manière à permettre l'implémentation facile d'un jeu de paramètres pour chaque concentration. Notamment, ont été décrites les variations de l'évolution du degré de saturation en fonction de la pression capillaire et de la perméabilité intrinsèque en fonction de la concentration. En premier lieu, la courbe de rétention pour chaque concentration en nitrate de cuivre (Fig. 5) a été modélisée par une fonction hyperbolique donnée par l'équation (5) :

$$
\mathrm{Sr}=\frac{1}{1+\mathrm{a} \sqrt{-\mathrm{PC}}}
$$

Un exemple de lissage de la courbe de rétention est donné en figure $8 \mathrm{a}$. La valeur du coefficient a été déterminée d'après les lissages; elle dépend de la concentration en cuivre et est présentée en figure 8b. En deuxième lieu, la perméabilité intrinsèque a été déduite des mesures de perméabilité illustrées en figure 4: l'évolution obtenue en fonction de la concentration en cuivre est représentée figure 9, telle qu'entrée dans le code de calcul. Bien que seules les concentrations de l'étude aient été considérées dans les simulations, d'autres concentrations peuvent ètre interpolées. A ce stade de l'étude du matériau Fo-Ca, une loi de variations de perméabilité relative de type $\mathrm{k}_{\mathrm{r}}=\mathrm{Sr}^{3}$, proposée par Alonso et al. (1999) a été implémentée dans le code en régime non saturé, en l'absence de résuitats expérimentaux permettant d'ajuster cette relation. Enfin, compte tenu des mesures effectuées le tableau II récapitule l'ensemble des paramètres d'entrée du modèle. Le fluide percolant dans le massif est assimilé à de l'eau de viscosité : $10^{-3} \mathrm{Pas}^{-1}$.

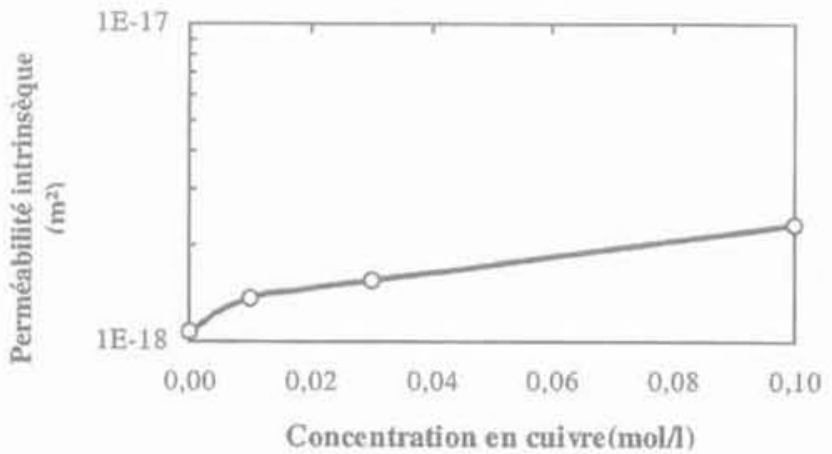

FiG. E Évolution de la perméabilité intrinsèque de la Fo-Ca en fonction de la concentration en cuivre. Evolution of the Fo-Ca intrinsic permeability versus copper concentration.

\section{2}

\section{Présentation des temps de transfert à l'échelle d'un massif de Fo-Ca pollué}

A ce stade, un maillage unidimensionnel, suffisant pour mettre en évidence des ordres de grandeur, a été réalisé. Une pression d'injection positive en paroi du modèle a été imposée sur une face, l'autre face étant libre de toute condition aux limites hydriques. Les prédictions ont été analysées en traçant l'évolution de la teneur en eau dans le domaine d'étude après différents temps d'imbibition. Les conditions aux limites choisies permettent d'aborder le problème à l'échelle d'un massif même si elles ne sont pas complètement représentatives du problème étudié.

Une première série de calculs a d'abord été réalisée à l'échelle des galettes de laboratoire. Les temps de saturation prédits étant proches de ceux obtenus lors des essais pour les différentes concentrations en nitrate de cuivre et les pressions d'injection de solutions imposées montrent la validité des lois d'évolutions des paramètres en fonction de la concentration en cuivre. Une deuxième série de calcul a porté sur l'étude d'un domaine de dimensions compatibles avec celles d'un stockage (longueur du domaine d'étude : 3 mètres) avec une pression d'injection en paroi plus faible, de $0,2 \mathrm{MPa}$. Les prédictions pour le massif sont présentées sur la figure 10 pour une période de 20 ans après différentes plages de temps écoulé. Les 
$[\mathrm{Cu}]=\mathbf{0}$
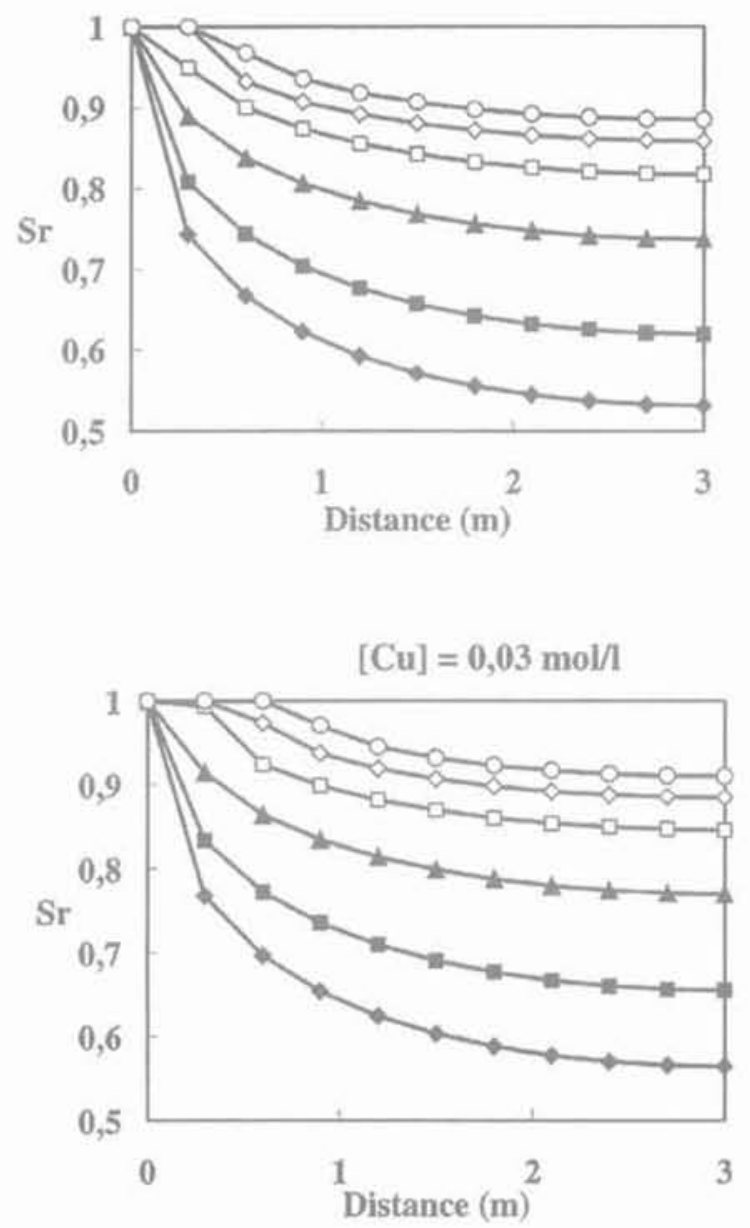
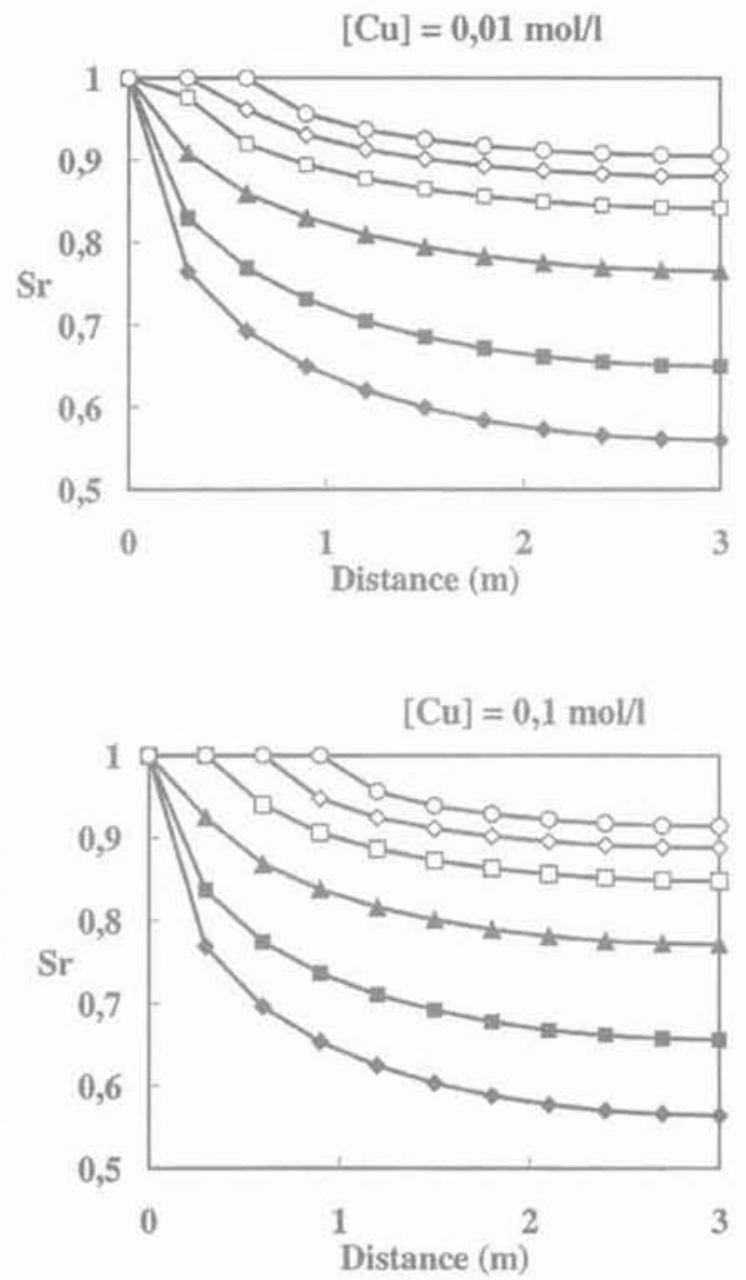
$: 1$ an
: 2 ans
$\mathbf{A}: 5$ ans
: 10 ans
$0: 15$ ans
$0: 20$ ans

FG.10 Simulations de la cinétique de saturation en eau à l'échelle d'un massif d'argile de $3 \mathrm{~m}$ de longueur après pollution avec des solutions de nitrate de cuivre.

Simulation of the water saturation kinetics trough a $3 \mathrm{mr}$ long clay polluted (with copper nitrate solutions) medium.

simulations montrent que la profondeur d'argile saturée après 20 ans est quatre fois plus grande dans le cas d'une pollution au nitrate de cuivre de concentration $0,1 \mathrm{~mol} / \mathrm{l}$ que dans le cas non pollué. De plus, l'accélération de la saturation est nette, y compris pour la concentration la plus faible de $0,01 \mathrm{~mol} / \mathrm{l}$. Ces résultats prédictifs reflètent, bien sûr, le choix d'un modèle de transfert simplifié mais aussi le fait que les évolutions des paramètres avec la concentration en polluant se cumulent:

- la sensibilité de ce type de modèle aux paramètres est telle que la perméabilité intrinsèque a une grande influence sur le volume entrant dans l'argile et donc sa cinétique de saturation ; c'est l'effet le plus important ; -l'évolution des propriétés de rétention après pollution agit dans le même sens.

Quant à la porosité, choisie constante dans le modèle, elle a en général, vu les plages de variations obtenues, moins d'influence dans ce type de modèle.
Ces résultats mettent en évidence l'utilité d'étudier les variations des paramètres de transfert de masse produites par la percolation de polluants car les conséquences à l'échelle d'un stockage peuvent être significatives. L'étude prédictive ci-dessus a été axée sur le transfert d'eau en fonction du temps dans un massif de Fo-Ca préalablement polluée au nitrate de cuivre, ce qui constitue un cas extrêmement sévère par rapport à une situation de stockage. Dans l'hypothèse où l'argile serait polluée au fur et à mesure de la saturation en lixiviat dans un stockage, le temps de transfert des polluants serait progressivement ralenti et l'évolution des propriétés de l'argile s'effectuerait en conséquence, ce qui donnerait lieu à des temps de saturation plus faibles que ceux prédits ici. Par ailleurs dans ce contexte, la viscosité du lixiviat devrait être, en toute rigueur, déterminée, bien qu'elle doive être, a priori, très peu différente de celle de l'eau. 


\section{Conclusion}

Ce travail avait pour objectif de déterminer l'efficacité de confinement, en terme de rétention de polluant, d'une argile gonflante compactée pouvant servir de barrière ouvragée dans un site de stockage. Parallèlement à des essais d'infiltration de solutions polluantes qui ont nécessité un développement technologique approprié, permettant ainsi de déterminer des paramètres nécessaires aux simulations, un code de calcul du transfert de masse en phase liquide a été utilisé pour prédire les durées de saturation en eau d'un massif pollué avec du nitrate de cuivre de différentes concentrations.

Les résultats expérimentaux obtenus ont montré qu'un échange cationique s'était produit et engendrait des modifications tant au niveau de la microstructure de l'argile (diminution de la distance basale), du volume de la phase poreuse que de son comportement macroscopique : gonflement, preméabilité et aptitude à la rétention, totale, en début d'infiltration. De plus, ces résultats ont permis de mettre en évidence que :

- les échanges cationiques sont indépendants des conditions d'injection du nitrate de cuivre sous forme soit d'une solution soit d'un mélange solution-gaz ; - même pour de fortes concentrations en polluant, l'aptitude au gonflement de la Fo-Ca n'est pas totalement inhibée :

- il existe simultanément deux processus chimiques qui contribuent à l'augmentation de la perméabilité :
- la dissolution de la calcite,

- les échanges ioniques entre les cations compensateurs de l'argile et le cuivre qui engendrent une diminution de l'espace interfoliaire sans modification du volume macroscopique ;

- les processus chimiques ont un effet significatif sur les propriétés de rétention du sol.

Pour simuler uniquement la réponse à l'infiltration d'eau dans un massif d'argile gonflante préalablement pollué avec des nitrates de cuivre, un jeu de paramètres correspondant à chaque concentration a été déterminé et un modèle simplifié de stockage utilisé. La présence du cuivre montre en première approximation, lors de la saturation en eau une forte augmentation de la perméabilité en présence de cuivre induisant une extension significative de la zone saturée du stockage, ce qui est relativement néfaste à son röle.

Enfin, bien que la perméabilité augmente fortement suite à une pollution, la Fo-Ca reste dans une gamme de perméabilité bien inférieure à celle d'un stockage de surface et il serait intéressant de transposer les résultats obtenus à ceux d'un stockage de déchets à grande profondeur.

\section{REMERCIEMENTS}

Ce travail a été réalisé avec le soutien tinancier de l'ANVAR et la collaboration d'élèves ingénieurs de I'ESEM. G. Biron, M. Fritzinger, J. Herbert. A. Lede, L. Massard et S. Thevenin, que les auteurs tiennent à remercier tout particulièrement.

\section{Bibliographie}

Alonso E.E., Vaunat J.. Gens A. - a Modelling the mechanical behaviour of expansive clays x. Engineering Geology, 54, 1999, p. 173-183.

Benzel W.M., Graf D.L - «Studies of smectite membrane behavior: importance of layer thickness and fabric in experiments at $20^{\circ} \mathrm{C}$. Geochim. Cosmochim. Acta, 48, 1984, p. 1769-1778.

Bradbury M.H., Baeyens B - $\alpha$ Modelling the sorption of $\mathrm{Zn}$ and $\mathrm{Ni}$ on $\mathrm{Ca}$-montmorilloniten. Geochim. Cosmochim. Acta, 63, 1999, p. 325-336.

Brandl H. - "Mineral liners for hazardous waste containment v. Geotechnique 42 (1) 1992 , p. $57-65$

Caillère S., Hènin S., Rautureau M. - Minéralogie des argiles. Masson. Paris, 1982.

Cases J.M., Berend 1., François M., Uriot J.P., Michot L.J., Thomas F. - $\alpha$ Mechanism of adsorption and desorption of water vapor by homoionic montmorillonite: 3 . The $\mathrm{Mq}^{2+}, \mathrm{Ca}^{2 *}, \mathrm{Sr}^{2+}$ and $\mathrm{Ba}^{2 *}$ exchanged forms w, Clays and Clay Minerals, vol. 45, 1997, p. 8-22.

Delattre-Louvel F, Lapeyre C., Struillou R. Arnould M. - «Retention mechanisms for toxic cations using artificial confinement barriers of clay-cement mix- tures n. Enaineering Geology, vol, 34. 1993, p. 151-158.

Hakkou R. Wahbi M. Pineau J.L. - "Analyse de la décharge de déchets urbains de Marrakech relativement à l'évolution de la régiementation marocaine en matière d'environnement . Science ef technique, $n^{\circ} 20,2000$, p. 12-18.

Jullien A. Lecomte P. - Dispositif perméa métrique à injecteur à pression contrôlée séparé. Brevet français ñ 00/07820. 2000.

Kozaki T., Sato H., Sato S., Ohashi H. "Diffusion mechanism of cesium ions in compacted montmorillonites. Engineering Geology, vol. 54, 1999, p. 223230.

Mertens D., Richelle B., Watelet M. " Audit technique de caractérisation d'une ancienne décharge : composition d'un lixiviat particulier n. TMS 6/95, 1995, p. 465-470.

Pothier C Jullien A. Pasquiou A. Robinet J.C. Plas F. - « Validation du code cléo par un essai sur maquette». $14^{*}$ Congrès français de mécanique. Toulouse, 1999.

Pothier C. - Etude des propriétés de confinement de matériaux argileux pour barrières ouvraqées de stockages de déchets. Thèse de doctorat de I'Université d'Orléans, 2001.

Pronost R., Matejka G. - u Les lixiviats de décharcjes d'ordures ménagères : production caractérisation traitement $p$. Environnement et technique, $\mathrm{n}^{2} 196$ 2000, p. $27-29$.

Rytwo G., Banin A.. Nir S. - - Exchange reactions in the $\mathrm{Ca}-\mathrm{Mg}$ - $\mathrm{Na}$-montmorillonite system v, Clays and Clay Minerals, vol. 44, $n^{2} 2,1996$, p. 276-285.

Shainberg I., Alperovitch N.I. Kerven R, "Charge density and Na-K-Ca exchange on smectites w. Clays and Clay Minerals, vol. $35, n^{\circ} 1,1987$, p. 68 73.

Singhal J.P. Khan S., Bansal O.P. - " Studies on the thermodynamics of exchange in clay s. Journal Inorg. Nucl. Chem. vol.38, 1976, p. 2297-2302.

Veniale F. $-\alpha$ The role of microfabric in clay soil stability n. Miner. Petrogr. Acta, 29A, 1985, p. 101-119.

Wu G. Li L. Y - «Modeling of heavy metal migration in sand/bentonite and the leachate $\mathrm{pH}$ effect .. Journal of Contaminant Hydrology 33, 1998, p. 313-336. 